\title{
Le Rire dans La Farce du cuvier
}

\author{
Frank Collins \\ (University of Toronto)
}

La Farce du cuvier est une courte pièce ( 348 vers) du quinzième siècle et dont le ressort principal c'est l'éternelle guerre qui oppose hommes et femmes, ici, surtout dans le context conjugal. ${ }^{1}$ Comme l'écrit l'auteur d'une édition critique récente de la pièce: "Au début la hiérarchie habituelle de la famille se trouve menacée par une femme autoritaire et une belle-mère importune. Cependant l'intervention du hasard et aussi la vivacité d'esprit d'un mari faible rétablissent finalement l'ordre traditionnel". 2 Ce résumé, que l'on peut caractériser d'interprétation "standard" en contexte pédagogique, est tout à fait conforme aux idées reçues pour ce qui est de l'interprétation de la farce en question. Et, c'est justement cette interprétation que je voudrais mettre tant soit peu en doute, en faveur d'une hypothèse tout autre.

Rappelons-nous l'essentiel de l'intrigue: Jacquinot, mari dominé par sa femme, se voit imposer par elle et par sa belle-mère, un "rollet", une liste de tous ses devoirs domicilaires. S'il ne les accomplit pas, il sera battu. Toutefois la femme, à un moment donné, en faisant la lessive, tombe dans un profond cuvier. Pour en sortir, il lui faudra l'aide de son mari -- intervention du hasard. Jacquinot profite de l'occasion, feint la naïveté, se réfère à son rollet sans y trouver l'obligation d'aider sa femme à se sauver du cuvier. - "Ce n'est pas à mon rollet" . . 3 La femme accepte que Jacquinot soit par la suite maître chez lui, que le rollet soit dechiré, et lui, en contrepartie, la sauve.

Au cours de la pièce, tout permet au lecteur ou au spectateur de croire que le programme narratif se constitue justement du rétablissement de l'ordre "normal" où c'est le mari qui règne en domicile. La femme dominante se trouve à un moment précis, vulnérable, impuissante -- lui la sauve, mais "à condition".

Voici, calquée sur le carré sémiotique, la transformation en question:

LittéRéalité, Vol. I, No. 1, Printemps/Spring 1989 


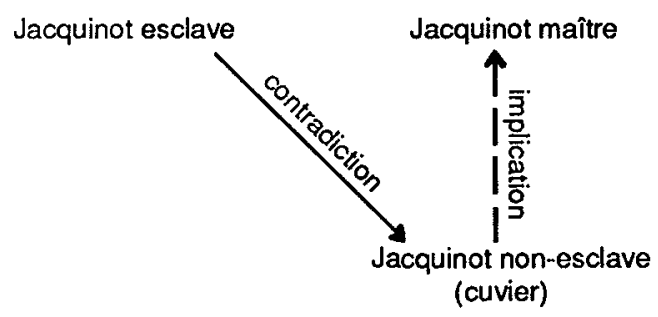

Revenons brièvement sur les relations du carré. On a un terme ("Jacquinot esclave"), on veut installer le contraire de cette situation ("Jacquinot maître"), et comme beaucoup l'on déjà dit, cela peut représenter un saut, une transformation trop brusque, résultat du seul caprice auctorial. Si, par contre, la destination finale ("Jacquinot maître") passe par l'installation de la simple contradiction du terme originel ("non-esclave") la relation logique l'implication peut entrer en jeu et, narrativement, mener à la situation finale voulue. Or, le hasard intervient, et Jacquinot se trouve puissant (le sujet acquiert la modalisation $\mathrm{du}$ /pouvoir-faire/), son aide est indispensable à la femme. Elle, pour se faire sauver, doit s'humilier. Ne s'agit-il pas pour lui d'une situation de non-esclave? Sûrement il n'est pas maître car il ne maîtrise pas sa vie, il n'est pas servi. Mais il n'est pas maîtrisé non plus -- il faut enfin, que la femme demande son aide, qu'elle assume le rôle de suppliante. Ainsi, de "non-esclave", on peut donc, suivant la relation logique d'implication", monter vers "Jacquinot maître", le contraire de "Jacquinot esclave". Voilà la pièce que les gens voient, paraît-il, si l'on se base sur les interprétations savantes. La critique, l'histoire littéraire, sont unanimes: l'intrigue de la Farce du cuvier c'est la transformation du mari dominé en mari dominant. Les paroles de Jacquinot à la fin de la pièce, annoncent que c'était par fantaisie que sa femme avait voulu le mettre "en subjection". Mais il nous dit que maintenant: "...Tous mes sens sont recovers"4 -- lui voit clair, sa femme est "ralliée", c'est-à-dire réconciliée, elle accepte, forcément, la nouvelle situation. Il s'agit là, selon toutes les interprétations, du mouvement narratif de cette farce.

Si l'on se représente ces transformations par l'entremise du schéma actantiel, l'on obtient le suivant: 


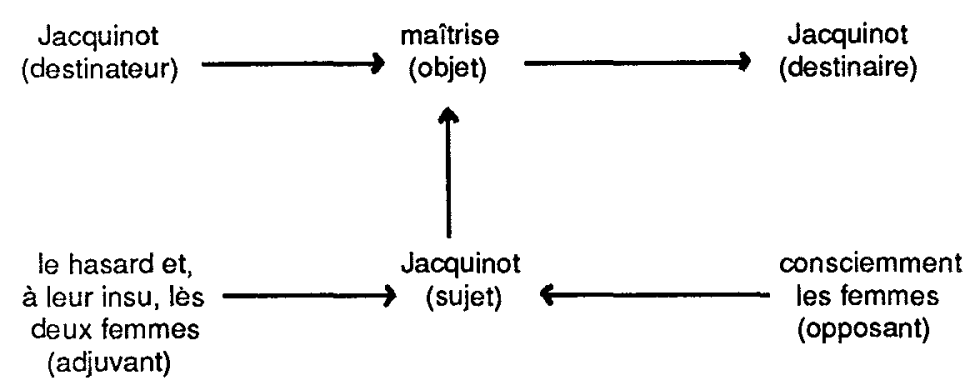

Le syncrétisme des rôles de Jacquinot se résume par ses paroles, "Je serai maistre en ma maison". 5

Le schéma souligne, en efffet, par l'importance du hasard, la non-initiative de Jacquinot dans le processus. Il sait profiter de l'occasion qui lui est proférée, il est habile, mais il est opportuniste, il n'est pas initiateur. Il se veut le rôle actantiel de destinateur, "Je serai maistre...", mais avant l'accident, c'est tout le contraire. Alors, encore une fois, voilà la pièce telle que décrite par les critiques -- Jacquinot se fait donner la maîtrise chez lui, et le mécanisme catalysateur c'est le hasard. Les mêmes critques décrivent le comique de cette farce comme étant très réussi, pas original, ce programme est éternel, mais il s'agit d'une pièce bien faite. Toutefois, quel comique? Mais le comique propre à la farce, aux fabliaux -- burlesque, violence sans conséquence, répétition, les victimes qui triomphent sur les bourreaux, grossièretés ou suggestion de grossièretés etc. Mais tout cela, tout le monde est d'accord, c'est un style, un ton, un divertissement véhiculant le thème : mari dominé ----> mari dominant.

En tant que lecteur ou spectateur, je ne perçois pas la pièce de cette façon, de même pour ce qui est du programme narratif. Quant à moi, ce qui est offert au lecteur c'est une offrande tout autre. C'est un texte qui s'inscrit dans le domaine de la blague, mais d'une blague très particulière, celle que Max Eastman 6 appelle the nonsense joke -- une blague qui pour son ressort essentiel, dans son inspiration essentielle, relève du nonsens imprévu et imprévisible. Elle dépend de l'introduction, à la fin d'une histoire ou d'une description d'une situation quelconque, d'un élément illogique qui fait tout écrouler. Le cerveau, l'esprit, nous dit Eastman, organise toutes les données reçues, par les cinq sens, et essaie constamment d'en faire quelque chose. 
L'esprit classe et ordonne les éléments d'un discours, d'un argument, d'une situation qui lui sont proposés, de façon à arriver à une conclusion qui en découle nécessairement. Or le nonsense joke, la blague illogique ou antilogique, fonctionne par la frustration de cette opération cérébrale instinctive. Ce genre de blague fournit des détails, des situations, des fragments d'arguments qui se proposent tout à fait comme éléments d'une éventuelle structure logique. Ils ne se trouvent pas sans pertinence réciproque. Mais, au dernier moment on $\mathrm{y}$ introduit un élément absurde, qui a l'air pertinent, mais qui fait tout éclater en empêchant toute possibilité de réalisation et /ou de clôture de la structure qui se formait. En d'autres termes, l'esprit est logiquement séduit, pour ensuite être brusquement frustré. Eastman nous dit qu'il s'agit là de l'équivalent mental du vilain tour comique physique, - du "practical joke", dirait-il, où la farce est jouée contre le corps de la victime : il lui est versé de l'eau sur la tête, une chaise bien preparée d'avance s'écroule, la victime tombe par terre, rien que dans l'accomplissement de mouvements corporels normaux et quotidiens - on s'assoit, on ouvre des portes etc.

La blague anti-logique fonctionne de la sorte, mais elle est jouée contre l'esprit organisateur qui, procédant normalement, décode ce qui lui est proposé avec la certitude de pouvoir en faire quelque chose. Brusquement, cette opération logique est irremédiablement arrêtée en cours de route, et on a un "accident provoqué", équivalent mental de la victimisation physique qui s'opère lors du "practical joke".

Des exemples : Deux hommes dans un bar à Toronto qui boivent tranquillement de la bière, et, à un moment donné, l'un dit à l'autre, "Est-ce que je vous ai connu, dans le temps, à New York?" L'autre qui répond, "Pas possible! J'ai jamais été à New York!" "Moi non plus", répond le premier, "c'est sans doute deux autres types".

Ou bien:

Un navire, un cargo, part de Hong Kong, chargé de yo yos. Le navire a sombré cent soixante-sept fois.

Encore une fois il s'agit bel et bien d'une séduction logique - il devrait y avoir une pertinence nécessaire entre le mouvement du jouet et celui d'un navire qui coule dans les profondeurs, remonte, redescend, remonte, etc. Mais il n'en est rien! Et le plaisir, le rire, pour ceux qui rient, est provoqué par l"impossibilité de penser logiquement, par le brusque et imprévu blocage de ce désir intellectuel humain de mettre de l'ordre, de clore le sens.

Revenons à nos moutons ( comme le dirait le juge dans la Farce de Maistre 
Pierre Pathelin ). Tout permet de croire, chez La Farce du cuvier, que l'on assiste à une transformation de Jacquinot dominé en Jacquinot dominant. Mais, si l'on y prête attention, on se rend compte que le même élément antilogique constaté pour ce qui concerne le nonsense joke intervient! La transformation de "non-esclave" en "maître" est bloquée, et tout se défait!

Par quoi? Vers la fin de la farce, au monent où Jacquinot tend la main vers sa femme pour lui permettre de sortir du cuvier, il nous déclare, "Je seray doncques desormais/ Maistre, puisque ma femme l'accorde". 7

L'anti-thèse "maître"/"ma femme l'accorde", nous rappelle Rousseau qui disait des Anglais qu'ils n'étaient libres que le jour des élections. Jacquinot n'est maître que momentanément et la femme liberée, qu'est-ce qu'il en sera? Maître parce que la femme lui donne permission de l'être, ce n'est pas vraiment être maître. La contradiction linguistique "maître parce que la femme dit d'accord, est ce qui signale la non-résolution de la contradiction situationnelle/conjugale (pour l'époque) de mari dominé. Mais le cornique essentiel de cette farce réside dans l'introduction imprévue et imprévisible à un moment clé du programme narratif mari dominé ------> mari dominant, de cet élément anti-programme -- dominant parce que la dominante est d'accord. L'attente de cette évolution vers une situation finale qui se promettait avec certitude, éclate, se défait, pour laisser place à la victoire de la contradiction, le triomphe de l'absurde. Étant donné cet actant/acteur clé (destinateur et destinataire) qu'est le hasard dans cette pièce, n'est pas là un mécanisme plus important que la simple transformation de mari dominé en mari dominant, proposée par la critique traditionnelle?

1 Le terme farce n'apparaît avec son sens technique que vers la fin du $14 \mathrm{e}$ siècle. Toutefois il s'agit là certainement d'un genre plus ancien. Les farces sont des pièces à longueur inégale -- 200 à 1500 vers -- qui s'inspirent des mêmes thèmes que l'on retrouve dans les fabliaux et dans les diverses branches du Roman de Renart : guerres conjugales, prêtres indignes, etc., et, surtout, le thème de trompeur trompé. Aucune classe sociale n'est épargnée dans ces pièces au rire tantôt complaisant, tantôt mordant.

2 Harden, A.R., ed., La Farce du cuvier. (New York: Appleton-CenturyCroft, 1967), p. 6. 
3 Harden, op. cit., v. 223, 235, 259, etc.

4 Harden, op. cit., v. 344.

5 Harden, op. cit., v. 306, 337.

6 Eastman, Max, The Enjoyment of Laughter. (New York: Simon and Shuster, 1936).

7 Harden, op. cit., v. 319-320. 